\title{
SARS-CoV-2 and myocardial injury: Few answers, many questions
}

\section{ABSTRACT}

Acute cardiac injury, defined as an elevated high-sensitivity troponin I or troponin T upon admission or during hospitalization, is common in patients with COVID-19, occurring in $10 \%$ to $35 \%$ of patients depending on the assay used and the population studied. Even though the mechanisms of SARS-CoV-2 myocardial injury are not well defined, type 1 myocardial infarction and fulminant myocarditis are rare. Often, acute cardiac injury occurs in patients with elevated inflammatory markers, and both are associated with worse outcomes. However, the extent to which treatments should differ for patients with acute cardiac injury, heightened systemic inflammation, or both, is unknown.

\section{KEY POINTS}

The mechanisms of acute cardiac injury in COVID-19 are still being defined but include oxygen supply-demand imbalance, microvascular and endothelial dysfunction, and micro- and macrothrombosis. In some patients, these manifestations may be driven by an inappropriate inflammatory response.

Like other patients, COVID-19 patients with ischemic STsegment elevation need emergency reperfusion therapy.

Patients with elevated troponin and elevated inflammatory markers may possibly benefit from immunosuppressive therapy, although further studies are needed.
T NITIAL CASE-FATALITY RATES in coronavi1 rus disease 2019 (COVID-19) have ranged from $2.3 \%$ to $7.3 \%, 1,2$ and given the burden of disease, the devastation is singularly alarming and unprecedented. Even though the predominant manifestations are respiratory, concomitant cardiovascular complications result in substantial morbidity and mortality. ${ }^{3}$

Acute cardiac injury in COVID-19 due to infection with SARS-CoV-2 has been defined primarily as an elevation in serum cardiac markers above the 99th percentile upper reference range, as it was in prior investigations of other viral infections, and the incidence has ranged from approximately $8 \%$ to $36 \%{ }^{4-8}$ Using the broad and inclusive definition of acute cardiac injury as an elevated high-sensitivity troponin I or troponin $\mathrm{T}$ upon admission or during hospitalization, the mortality rate has been striking-over 50\% in initial reports.,

Given this startling signal, amid our everchanging understanding of this pandemic, the following questions warrant emphasis:

- What is the mechanism of SARS-CoV-2associated myocardial injury?

- To what extent are SARS-CoV-2 patients with myocardial injury a distinct population?

- What are possible treatment options for myocardial injury associated with SARSCoV-2 infection?

\section{WHAT IS THE MECHANISM?}

With regard to mechanism, the primary question is whether SARS-CoV-2 infection precipitates myocardial infarction with an oxygen supply-demand imbalance, either with or without acute coronary plaque pathology (type 1 and 2 myocardial infarction), or conversely, 
causes myocardial injury mediated by the virus itself or the cytokine response to it.

Viral infections are well known to lead to adverse cardiovascular events, either by increased metabolic demand in the setting of limited cardiac reserve or by precipitating plaque rupture in the setting of inflammation and a prothrombotic state. ${ }^{9}$ Of note, influenza vaccination has been shown to reduce hospitalizations for cardiac disease. ${ }^{10}$ In addition, certain viruses (eg, parvovirus B19 and influenza) commonly cause myocarditis.

Although myocardial injury has not been prominent with other coronaviruses, unfortunately, SARS-CoV-2 appears to be behaving differently. Despite an overall case-fatality rate of approximately $10 \%$ in symptomatic patients in the previous SARS-CoV outbreak that resulted in severe acute respiratory syndrome (SARS), cardiac complications were anecdotal and limited to case reports or series. ${ }^{11}$ Similarly, despite an even higher case-fatality rate in Middle East respiratory syndrome due to MERS-CoV, cardiac complications were limited. ${ }^{9,12}$

In contrast, in an initial report of causes of death in COVID-19, one-third were con-

Myocardial injury has not been prominent with other coronaviruses, but SARS-CoV-2 appears to be behaving differently sidered secondary to respiratory failure with myocardial damage, and nearly another tenth were considered secondary to myocardial damage alone. ${ }^{13}$ Furthermore, perimyocarditis from SARS-CoV-2 infection has been reported in the absence of symptomatic respiratory disease, ${ }^{14}$ though fulminant myocarditis-generally defined as sudden and severe inflammation of the myocardium resulting in myocyte necrosis, edema, and cardiogenic shock - seems to be a rare presentation with SARS-CoV-2.

Of note, SARS-CoV-2 enters respiratory and cardiac cells via angiotensin-converting enzyme 2 (ACE 2), a membrane-bound protein. ${ }^{3,9}$ Yet this potential cardiac tropism offers an incomplete explanation for the seemingly disproportionate cardiac manifestations of COVID-19, given that SARS-CoV also uses ACE 2 as a functional receptor. ${ }^{15,16}$

Alternatively, myocardial injury may be exacerbated by an inappropriate activation of type 1 T-helper cells and cell-mediated immunity with associated cytokine storm. ${ }^{3}$ A recent autopsy study ${ }^{17}$ is consistent with this hypothesis. Among 39 patients, SARS-CoV-2 cardiac infection was documented in $61.5 \%$, and patients with higher viral loads had greater expression of proinflammatory genes. However, inflammatory cell infiltrates typical of active myocarditis were not observed.

\section{A DISTINCT POPULATION?}

These putative mechanisms of injury are integrally entwined with the second question of whether patients with SARS-CoV-2-associated myocardial injury represent a distinct population. For one, COVID-19 patients with elevated troponins are older and have more cardiovascular comorbidities, such as coronary artery disease, chronic heart failure, hypertension, and diabetes mellitus. ${ }^{5,6,8}$ These findings support the concept of myocardial oxygen supply-demand mismatch with resultant ischemia in a vulnerable population.

However, in patients who succumb to COVID-19, troponin levels may continue to rise throughout the illness, a pattern distinct from the typical rise and fall after an ischemic insult. ${ }^{6}$ Moreover, about a third of patients may demonstrate an increase in troponin over time, and these patients have a higher mortality rate. ${ }^{8}$ Importantly, patients with elevated troponins have higher levels of inflammatory markers such as C-reactive protein (CRP). ${ }^{8}$ The increases in troponin and CRP appear to parallel each other, and the overall correlation is similar in magnitude to the correlation between troponin and $\mathrm{N}$-terminal probrain natriuretic peptides. These observations, though nascent, suggest that some patients may develop a hyperinflammatory state that perpetuates nonischemic myocardial injury.

Given that elevated troponin is associated with a high mortality rate and that the mechanism of injury could be related to increased systemic inflammation, as more data are emerging, consideration should be given to checking troponin upon admission, with surveillance testing during the initial days of hospitalization. Further considerations in this initial clinical approach include assessing cardiac risk factors and the magnitude of the inflammatory response.

A rise and fall of cardiac markers in the presence of signs and symptoms of myocar- 
dial ischemia, such as new ischemic changes on electrocardiography or imaging evidence of regional myocardial dysfunction in a pattern compatible with ischemia, diagnoses a myocardial infarction. The absence of these features defines myocardial injury, and substantial elevations in CRP may point toward cytokine-mediated damage.

Traditionally, cardiac imaging would feature prominently in the distinction between acute myocardial infarction and injury. Given limited resources and the need to minimize exposure to COVID-19 patients, this decision will be individualized, though it will involve selective use of focused echocardiography.

In patients who have convalesced from COVID-19, studies have shown that myocardial damage and inflammation may be evident in a majority of patients when assessed with cardiac magnetic resonance imaging. ${ }^{18,19} \mathrm{How}$ ever, the cross-sectional design of these studies precludes any assessment of causality, and the clinical implications are unclear. Therefore, in the absence of another indication, cardiac magnetic resonance imaging is currently not clinically recommended in asymptomatic patients who have recovered from COVID-19.

Of note, the overlap between acute myocardial infarction, myocardial injury, and heightened systemic inflammation continues to be defined, though these considerations do aid in risk stratification. An elderly patient with coronary artery disease, diabetes mellitus, and elevations in troponin and CRP will have among the poorest prognoses. However, even in the absence of these risk factors, a patient with elevated troponin and inflammatory markers is at increased risk. ${ }^{8}$ In evaluating patients with an elevated troponin, we are well accustomed to risk stratification according to cardiovascular comorbidities, but with $\mathrm{CO}$ VID-19, we should also risk-stratify based on the degree of heightened inflammation.

\section{WHAT ARE THE POSSIBLE TREATMENTS?}

Finally, the consideration of treatment options in a patient with a positive troponin test is informed by the presumed answers to the first 2 questions. Specifically, does the mechanism of injury seem more likely related to myocardial infarction with oxygen supply- demand mismatch, or to direct myocardial injury? And is this a patient with underlying cardiac conditions, increased systemic inflammation, or both?

Treatment strategies for type 1 myocardial infarction are well delineated, and treatment of type 2 myocardial infarction includes addressing the underlying cause and providing therapies to improve the myocardial oxygen supply-demand mismatch, especially in the setting of known fixed coronary stenosis. Importantly, therapies such as beta-blockers and vasodilators must be used judiciously to avoid precipitating decompensated heart failure or shock. In this setting, revascularization is rarely indicated, and the benefit of antiplatelet and anticoagulant therapy is unknown.

With COVID-19, treatment is supportive, and directed therapies are urgently required. Remdesivir has been shown to shorten the time to recovery, and in hospitalized patients who are hypoxic, dexamethasone improves survival. ${ }^{20}$ In severe disease, the success of dexamethasone suggests that morbidity and mortality may be driven by heightened inflammation. ${ }^{21,22}$ This increased inflammation may progress to secondary hemophagocytic lymphohistiocystosis and resultant fatal hypercytokinemia with multiorgan failure. In this inflammatory state, further immunosuppression may improve outcomes.

In cardiac disease, promising immune treatments target autoinflammation, a process driven by endogenous danger signals and perpetuated by inflammasome-induced cytokine production. Such therapies have demonstrated efficacy and include colchicine, rilonacept, and anakinra in pericarditis and colchicine and canakinumab in atherosclerotic disease. ${ }^{23-27}$ Colchicine inhibits tubulin polymerization and inflammasome activity, whereas anakinra, rilonacept, and canakinumab inhibit interleukin 1, a cytokine that is central to the inappropriate innate immune response. In open-label case-control studies, anakinra ${ }^{28}$ and canakinumab ${ }^{29}$ have shown promise in treating severe COVID-19 pneumonia, and a small randomized trial suggested a potential benefit of colchicine. ${ }^{30}$ Accordingly, larger randomized studies with these therapies are currently enrolling patients.

\section{Even without other risk factors, a patient with elevated troponin and inflammatory markers is at increased risk}




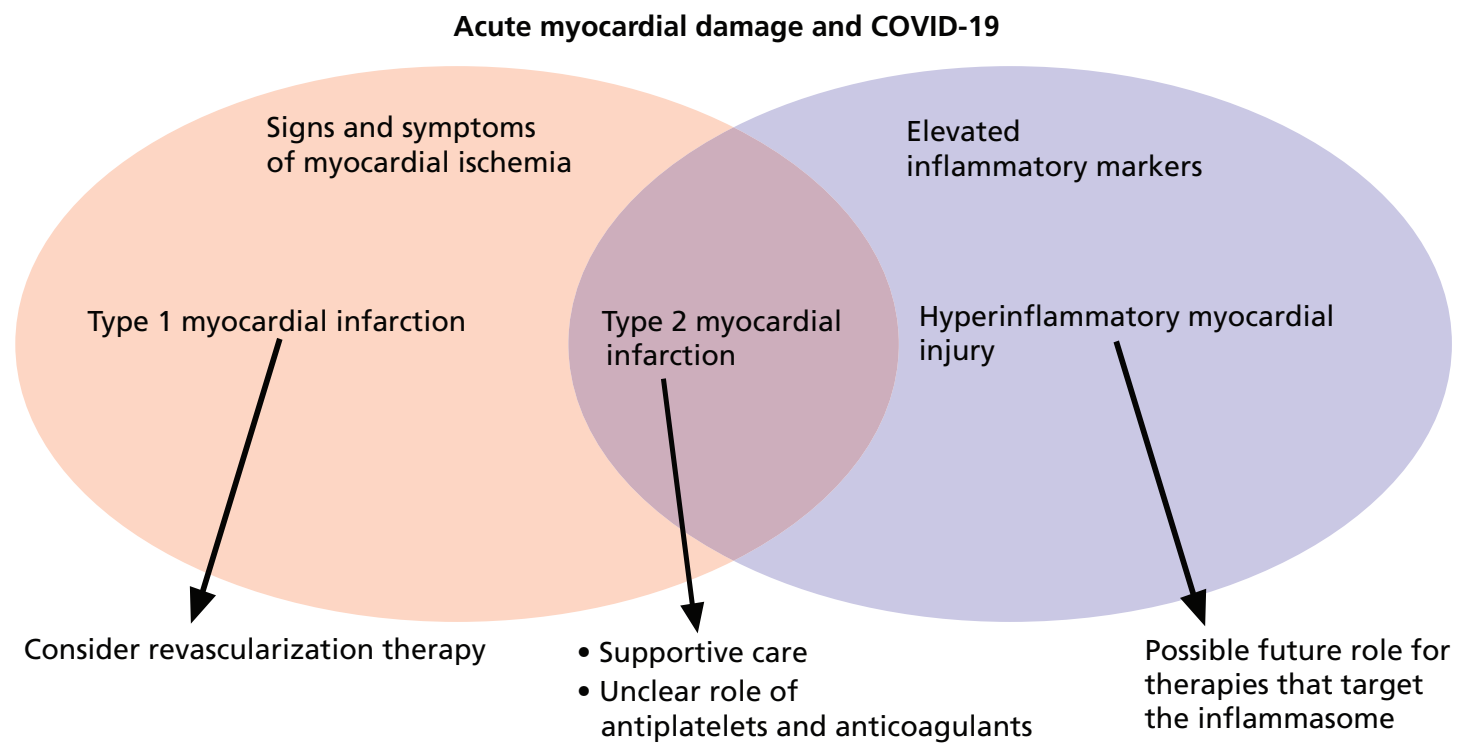

Figure 1. Three broad causes of acute cardiac injury.

\section{FEW ANSWERS, MANY QUESTIONS}

For troponin-positive COVID-19 patients, we currently have few answers and many questions. With COVID-19, typical acute coronary syndromes and classic myocarditis occur rarely. The mechanisms of acute cardiac injury are still being defined, but include oxygen supply-demand imbalance, microvascular and endothelial dysfunction, as well as microand macrothrombosis. In some patients, these manifestations may be driven by an inappropriate inflammatory response.

\section{REFERENCES}

1. Wu Z, McGoogan JM. Characteristics of and important lessons from the coronavirus disease 2019 (COVID-2019) outbreak in China: summary of a report of 72,314 cases from the Chinese Center for Disease Control and Prevention. JAMA 2020; Feb 24. doi:10.1001/jama.2020.2648

2. Onder G, Rezza G, Brusaferro S. Case-fatality rate and characteristics of patients dying in relation to COVID-19 in Italy. JAMA 2020; Mar 23. doi:10.1001/jama.2020.4683

3. Zheng Y-Y, Ma Y-T, Zhang J-Y, Xie X. COVID-19 and the cardiovascular system. Nat Rev Cardiol 2020; 17(5):259-260. doi:10.1038/s41569-020-0360-5

4. Wang D, Hu B, Hu C, et al. Clinical characteristics of 138 hospitalized patients with 2019 novel coronavirus-infected pneumonia in Wuhan, China. JAMA 2020; 323(1):1061-1069. doi:10.1001/jama.2020.1585

5. Shi S, Qin M, Shen B, et al. Association of cardiac injury with mortality in hospitalized patients with COVID-19 in Wuhan, China. JAMA Cardiol 2020; 5(7):802-810. doi:10.1001/jamacardio.2020.0950

6. Guo T, Fan Y, Chen M, et al. Cardiovascular implications of fatal outcomes of patients with coronavirus disease 2019 (COVID-19). JAMA Cardiol 2020; 5(7):1-8. doi:10.1001/jamacardio.2020.1017

7. Huang C, Wang Y, Li X, et al. Clinical features of patients infected
In general, for the practicing clinician, we can consider 3 broad categories of patients with COVID-19 and abnormal troponins:

- Patients with ischemic ST elevation, who need emergency reperfusion therapy

- Patients with troponin elevation without systemic heightened inflammation, who need supportive care

- Patients with elevated troponin and inflammatory markers, who may possibly benefit from immunosuppressive therapy, although further studies are needed (Figure 1).

with 2019 novel coronavirus in Wuhan, China. Lancet 2020; 395(10223):497-506. doi:10.1016/S0140-6736(20)30183-5

8. Lala A, Johnson KW, Januzzi JL, et al. Prevalence and impact of myocardial injury in patients hospitalized with COVID-19 infection. J Am Coll Cardiol 2020; 76(5):533-546. doi:10.1016/j.jacc.2020.06.007

9. Xiong TY, Redwood S, Prendergast B, Chen M. Coronaviruses and the cardiovascular system: acute and long-term implications. Eur Heart J 2020; Mar 18. doi:10.1093/eurheartj/ehaa231

10. Nichol KL, Nordin J, Mullooly J, Lask R, Fillbrandt K, Iwane M. Influenza vaccination and reduction in hospitalization for cardiac disease and stroke among the elderly. N Engl J Med 2003; 348(14):13221332. doi:10.1056/NEJMoa025028

11. Chong PY, Chui P, Ling AE, et al. Analysis of deaths during the severe acute respiratory syndrome (SARS) epidemic in Singapore: challenges in determining a SARS diagnosis. Arch Pathol Lab Med 2004; 128(2):195-204. doi:10.1043/1543-2165(2004)128<195:AODD TS>2.0.CO;2

12. Assiri A, McGreer A, Perl TM, et al; KSA MERS-CoV Investigation Team. Hospital outbreak of Middle East respiratory syndrome coronavirus. N Engl J Med 2013; 369(5):407-416. doi:10.1056/NEJMoa1306742

13. Ruan Q, Yang K, Wang W, Jiang L, Song J. Clinical predictors of mortality due to COVID-19 based on an analysis of data of 150 pa- 
tients from Wuhan, China. Intensive Care Med 2020; 46(5):846-848. doi:10.1007/134-020-05991-x

14. Inciardi RM, Lupi L, Zaccone G, et al. Cardiac involvement in a patient with coronavirus disease 2019 (COVID-19). JAMA Cardiol 2020; 5(7):1-6. doi:10.1001/jamacardio.2020.1096

15. Li W, Moore MJ, Vasilieva N, et al. Angiotensin-converting enzyme 2 is a functional receptor for the SARS coronavirus. Nature 2003; 426(6965):450-454. doi:10.1038/nature02145

16. Vaduganathan M, Vardeny $P$, Michel T, McMurray JJV, Pfeffer MA, Solomon SD. Renin-angiotensin-aldosterone system inhibitors in patients with COVID-19. N Engl J Med 2020; Mar 30. doi:10.1056/NEJMsr2005760

17. Linder D, Fitzek A, Brauninger $\mathbf{H}$, et al. Association of cardiac infection with SARS-CoV-2 in confirmed COVID-19 autopsy cases. JAMA Cardiol 2020; Jul 27. doi:10.1001/jamacardio.2020.3551

18. Knight DS, Kotecha T, Razvi Y, et al. COVID-19: Myocardial injury in survivors. Circulation 2020; Jul 14. doi:10.1161/CIRCULATIONAHA.120.049252

19. Puntman VO, Carerj L, Wieters I, et al. Outcomes of cardiovascular magnetic resonance imaging in patients recently recovered from COVID-19. JAMA Cardiol 2020 Jul 27. doi:10.1001/jamacardio.2020.3557

20. Beigel JH, Tomashek KM, Dodd LE, et al. Remdesivir for the treatment of Covid-19-preliminary report. N Engl J Med 2020; May 22. doi:10.1056/NEJMoa2007764

21. Mehta P, McAuley DF, Brown M, Sanchez E, Tattersall RS, Manson JJ; HLH Across Specialty Collaboration, UK. COVID-19: consider cytokine storm syndromes and immunosuppression. Lancet 2020; 395(10229):1033-1034. doi:10.1016/S0140-6736(20)30628-0

22. RECOVERY Collaborative Group; Horby P, Lim WS, Emberson JR, et al. Dexamethasone in hospitalized patients with Covid-19-preliminary report. N Engl J Med 2020 jul 17. doi:10.1056/NEJMoa2921436

23. Imazio M, Belli R, Brucato R, et al. Efficacy and safety of colchicine for treatment of multiple recurrences of pericarditis (CORP-2): a multicentre, double-blind, placebo-controlled randmised trial. Lancet 2014; 383(9936):2232-2237. doi:10.1016/S0140-6736(13)62709-9

24. Brucato A, Imazio M, Gattorno M, et al. Effect of anakinra on recurrent pericarditis among patients with colchicine resistance and corticosteroid dependence: the AIRTRIP randomized clinical trial. JAMA 2016; 316(18):1906-1912. doi:10.1001/jama.2016.15826

25. Tardif JC, Kouz S, Waters DD, et al. Efficacy and safety of lowdose colchicine after myocardial infarction. N Engl J Med 2019; 381(26):2497-2505. doi:10.1056/NEJMoa1912388

26. Ridker PM, Everett BM, Thuren T, et al; CANTOS Trial Group. Antiinflammatory therapy with canakinumab for atherosclerotic disease. N Engl J Med 2017; 377(12):1119-1131. doi:10.1056/NEJMoa1707914

27. Klein A, Imazio M, Brucato A, et al. RHAPSODY: rationale for and design of a pivotal phase 3 trial to assess efficacy and safety of rilonacept, an IL-1a and IL-1b trap, in patients with recurrent pericarditis. Am Heart J 2020 Jul 14. doi:10.1016/j.ahj.2020.07.004

28. Cavalli G, De Luca G, Campochiaro C, et al. Interleukin-1 blockade with high-dose anakinra in patients with COVID-19, acute respiratory distress syndrome, and hyperinflammation: a retrospective cohort study. Lancet Rheumatol 2020 2(6):e325-e331. doi:10.1016/S2665-9913(20)30127-2

29. Ucciferri C, Auricchio A, Di Nicola M, et al. Canakinumab in a subgroup of patients with COVID-19. Lancet Rheumatol 2020; 2(8):e457-e458. doi:10.1016/S2665-9913(20)30167-3

30. Deftereos SG, Giannopoulos G, Vrachatis DA, et al. Effect of colchicine vs standard care on cardiac and inflammatory biomarkers and clinical outcomes in patients hospitalized with coronavirus disease 2019: the GRECCO-19 Randomized Clinical Trial. JAMA Netw Open 2020; 3(6):e2013136. doi:10.1001/jamanetworkopen.2020.13136

Address: Paul Cremer, MD, Cardiovascular Medicine, J1-5, Cleveland Clinic, 9500 Euclid Avenue, Cleveland, $\mathrm{OH}$ 44195; cremerp@ccf.org 